\section{Confiabilidade teste-reteste do Índice de Capacidade para o Trabalho (ICT) em trabalhadores de enfermagem}

\section{Test-retest reliability of the Work Ability Index (WAI) in nursing workers}

\section{Resumo}

Este artigo avalia a confiabilidade teste-reteste do Índice de Capacidade para o Trabalho (ICT) em trabalhadores de enfermagem. Foi aplicado questionário autopreenchível duas vezes a um grupo de 80 trabalhadores de enfermagem (enfermeiros, técnicos e auxiliares de enfermagem) de um hospital público no Município do Rio de Janeiro, com intervalo entre sete e quinze dias. A confiabilidade foi estimada pela estatística kappa ponderada quadrática, pelo coeficiente de correlação intraclasse (CCI) e pelo gráfico de Bland e Altman. Dos participantes, $81 \%$ eram mulheres e com idade variando de 22 a 67 anos (média = 39,$1 ; \mathrm{DP}=10,8$ anos) e $36,3 \%$ tinham ensino superior completo. O escore global do ICT apresentou $\mathrm{CCI}=0,79\left(\mathrm{IC}_{95 \%} 0,67\right.$ a 0,86$) \mathrm{e}$ kappa $=0,69\left(\mathrm{IC}_{95 \%} 0,50\right.$ a 0,80) para o ICT categórico (classificado em baixo, moderado, bom e ótimo). O kappa dos itens do ICT variou de 0,39 a 0,82 e o gráfico de Bland e Altman não mostrou um padrão sistemático. A concordância entre as medidas de teste-reteste indica grau aceitável de confiabilidade, sugerindo adequação do processo de aferição entre trabalhadores de enfermagem.

Palavras-chave: Confiabilidade. Capacidade para o trabalho. Enfermagem. Kappa. Coeficiente de Correlação Intraclasse. Gráfico de Bland e Altman.
Sérgio Henrique Almeida da Silva Junior'

Ana Glória Godoi Vasconcelos'

Rosane Harter Griep"

\section{Lúcia Rotenberg"}

' Escola Nacional de Saúde Pública Sérgio Arouca - ENSP/FIOCRUZ, Rio de Janeiro, RJ.

" Instituto Oswaldo Cruz - IOC/FIOCRUZ, Rio de Janeiro, RJ. 


\section{Abstract}

This paper assesses the test retest reliability of the Work Ability Index (WAI) in nursing workers. A self-administered questionnaire was applied twice to a group of 80 workers (nurses and nursing aides/assistants) at a public hospital in Rio de Janeiro, Brazil within an interval from seven to fifteen days. The reliability was estimated using quadratic weighted kappa statistics, interclass correlation coefficient (ICC) and the Bland and Altman plot. Eighty-one percent of participants were women aged betweenfrom 22 to 67 years (mean $=39.1 ; \mathrm{SD}=10.8$ years); $36.3 \%$ had completed higher education. The global score of the WAI presented ICC= 0.79 (IC95\% 0.67 to 0.86 ) and weighted kap$\mathrm{pa}=0.69$ (CI95\% 0.50 to 0.80 ) for categorical WAI (classified as low, moderate, good and excellent). The quadratic weighted kappa of the WAI items ranged from 0.39 to 0.82 and the Bland and Altman plot did not show a systematic pattern. The agreement between the test and retest measures shows an acceptable degree of reliability, suggesting the adequacy of the assessment process among nursing workers.

Keywords: Reliability. Work ability. Nursing. Kappa. Interclass Correlation Coefficient (ICC). Bland and Altman graphic.

\section{Introdução}

O Índice de Capacidade para o Trabalho (ICT) avalia a percepção do trabalhador em relação a “o quão bem está, ou estará, neste momento ou num futuro próximo, $\mathrm{e}$ o quão bem ele pode executar seu trabalho, em função das exigências, de seu estado de saúde e capacidades físicas e mentais" ${ }^{1}$. É considerada uma medida preditiva de situações de perda de capacidade laboral, aposentadoria precoce, absenteísmo por doença e desemprego ${ }^{2}$.

No Brasil, o ICT tem sido utilizado para avaliar a capacidade funcional e/ou identificar fatores associados entre trabalhadores da indústria ${ }^{3-6}$, eletricitários ${ }^{7}$, docentes universitários $^{8}$, motoristas de ônibus ${ }^{9} \mathrm{e}$ equipes de enfermagem ${ }^{10-14}$. No entanto, apenas três estudos avaliaram o desempenho psicométrico deste índice ${ }^{3,14,15} \mathrm{e}$ apenas um deles abrangeu profissionais da saúde, especificamente equipes de enfermagem.

O interesse no estudo do ICT em profissionais da enfermagem se deve a peculiaridades deste grupo no Brasil, como os plantões longos (geralmente de 12 horas) e o acúmulo de empregos, o que gera jornada semanal extensa à qual se soma o trabalho doméstico, por se tratar de um grupo majoritariamente feminino. No contexto hospitalar, a enfermagem é a maior força de trabalho. Nesse local, os trabalhadores são expostos a diversos estressores ocupacionais, sejam eles ambientais ou organizacionais, que configuram exigências e cargas de trabalho muito específicas e potencialmente determinantes de comprometimento da saúde, bem estar e capacidade para o trabalho, conforme já demonstrado em estudos nacionais ${ }^{10-13,16} \mathrm{e}$ internacionais ${ }^{17}$.

Estudos epidemiológicos fornecem evidências sobre determinantes de doenças em populações humanas que dependem, entre outros condicionantes, da qualidade das medidas, exames e dados de entrevistas, avaliados através de estudos de validade e confiabilidade. O presente estudo avalia a confiabilidade teste-reteste (estabilidade 
temporal) da versão brasileira do ICT entre trabalhadores de enfermagem.

\section{Métodos}

A coleta de dados do estudo foi realizada em um hospital público na cidade do Rio de Janeiro, entre abril e maio de 2005. Para a operacionalização do estudo teste-reteste foi selecionada uma amostra sistemática de $10 \%$ de uma listagem de 1.100 trabalhadores da assistência de enfermagem, que incluía enfermeiros, técnicos e auxiliares de enfermagem de plantões noturnos e diurnos. Adotou-se a estratégia de aplicação do questionário autopreenchível durante o horário de trabalho após a leitura e assinatura do termo de consentimento, em locais reservados e com o apoio de aplicadores treinados. Para testar a adequação do processo de aferição do instrumento, solicitou-se que os respondentes preenchessem novamente o questionário com intervalo entre sete e quinze dias. Dos 111 trabalhadores que participaram do teste, $80(72,1 \%)$ aderiram também ao reteste; não foram identificadas perdas seletivas no que se refere a características sociodemográficas e ocupacionais. Situações de faltas, trocas ou impossibilidade de responder naquela oportunidade demandavam uma abordagem três dias após, em função da organização do trabalho em plantões a cada três dias. Assim, quatro trabalhadores (5\%) responderam ao reteste com intervalo maior que o planejado (18 ou 19 dias). O estudo não envolveu conflitos de interesse e foi aprovado pelo Comitê de Ética em Pesquisa da Fundação Oswaldo Cruz - FIOCRUZ (Protocolo: 241/04).

\section{O Índice de Capacidade para o Trabalho}

Utilizou-se versão do ICT traduzida e adaptada para o Português brasileiro publicada por Tuomi et al. ${ }^{1}$, validada por Martinez et al. ${ }^{3}$ e Silva Junior et al. ${ }^{14}$. Os itens componentes do ICT, sintetizados em sete dimensões, estão apresentados no Quadro 1.

Quadro 1 - Número de questões e pontos dos escores de cada dimensão do ICT.

Chart 1 - Number of questions and points scores for each dimension of the WAI.

\begin{tabular}{|c|c|c|}
\hline Item & $\begin{array}{l}\text { No de } \\
\text { questões }\end{array}$ & Número de pontos (escores) das respostas \\
\hline $\begin{array}{l}\text { 1. Capacidade para o trabalho comparada com a } \\
\text { melhor de toda vida. }\end{array}$ & 1 & 0-10 pontos (Valor assinalado no questionário) \\
\hline $\begin{array}{l}\text { 2. Capacidade para o trabalho em relação a } \\
\text { exigências físicas. }\end{array}$ & 2 & $\begin{array}{l}\text { Número de pontos ponderados de acordo com a } \\
\text { natureza do trabalho. }\end{array}$ \\
\hline $\begin{array}{l}\text { 3. Número de doenças atuais diagnosticadas } \\
\text { pelo médico. }\end{array}$ & $\begin{array}{c}1 \\
\text { (Lista de } 56 \\
\text { Doenças) }\end{array}$ & $\begin{array}{l}\text { Pelo menos } 5 \text { doenças }=1 \text { ponto. } \\
4 \text { doenças }=2 \text { pontos } \\
3 \text { doenças }=3 \text { pontos } \\
2 \text { doenças }=2 \text { pontos } \\
1 \text { doença }=5 \text { pontos } \\
\text { Nenhuma doença }=7 \text { pontos }\end{array}$ \\
\hline $\begin{array}{l}\text { 4. Perda estimada para o trabalho por causa de } \\
\text { doenças. }\end{array}$ & 1 & $\begin{array}{l}\text { 1-6 pontos (valor circulado no questionário; o pior } \\
\text { valor escolhido) }\end{array}$ \\
\hline $\begin{array}{l}\text { 5. Faltas ao trabalho por doenças no último ano } \\
\text { (12 meses). }\end{array}$ & 1 & 1-5 pontos (valor circulado no questionário). \\
\hline $\begin{array}{l}\text { 6. Prognóstico próprio da capacidade para o } \\
\text { trabalho daqui a } 2 \text { anos. }\end{array}$ & 1 & 1, 4 ou 7 pontos (valor circulado no questionário). \\
\hline 7. Recursos mentais. & 3 & $\begin{array}{l}\text { Os pontos das questões são somados e o resultado } \\
\text { é contado da seguinte forma: } \\
\text { Soma } 0-3=1 \text { ponto. } \\
\text { Soma } 4-6=2 \text { pontos. } \\
\text { Soma } 7-9=3 \text { pontos. } \\
\text { Soma } 10-12=4 \text { pontos. }\end{array}$ \\
\hline
\end{tabular}

Fonte/Source:Tuomi et al (2005) 
O ICT global corresponde a um escore que varia de 7 (pior índice) a 49 (melhor índice), que tem sido categorizado em quatro níveis: baixo (7-27), moderado (28-36), bom (37-43) e ótimo (44-49) ${ }^{1}$.

\section{Análises dos dados}

Para a análise de estabilidade teste-reteste dos itens, dos escores das dimensões (variáveis contínuas) e do escore total do ICT foi utilizado o coeficiente de correlação intraclasse (CCI). Na avaliação de variáveis ordinais com mais de duas categorias foi aplicada a estatística kappa com ponderação quadrática. As respostas discordantes foram ponderadas pelos quadrados dos desvios da concordância exata ${ }^{18}$, por possibilitar interpretação equivalente ao CCI.

Para todas as estatísticas foram estimados intervalos de $95 \%$ de confiança; para avaliação do Kappa adotaram-se os critérios propostos por Landis e Koch ${ }^{19}$ para interpretação do grau de concordância: a) quase perfeita: 0,81 a 1,00; b) forte: 0,61 a 0,80 ; c) moderada: 0,41 a 0,60 ; d) regular: 0,21 a 0,40 ; d) discreta: 0 a 0,20 ; e) pobre < 0. Para avaliação do CCI foram utilizados os seguintes critérios: a) alta: 1 a 0,75 ; b) moderada 0,4 a 0,$74 ;$ c) fraca $<0,4(18)$. Na avaliação do padrão das discordâncias entre medidas repetidas (teste-reteste), foi utilizado o gráfico de Bland e Altman ${ }^{20}$.

No caso de indivíduos que apresentassem menos de $50 \%$ de dados perdidos ( 5 ou menos itens sem resposta no questionário ICT), adotou-se a imputação dos dados perdidos ${ }^{21}$ utilizando o valor médio se a escala fosse contínua, ou mediana se a escala fosse discreta.

A normalidade do escore total do ICT foi testada através do teste KolmogorovSmirnov e a comparação das médias dos escores totais do ICT no teste e no reteste foi feita através do teste t pareado.

\section{Resultados}

Foram entrevistados trabalhadores de vinte e sete setores do hospital, sendo $81,3 \%$ mulheres e $36,3 \%$ com ensino superior completo. A idade variou de 22 a 67 anos; a média foi 39,1 anos [DP = 10,8]. Em relação às características ocupacionais, $30 \%$ eram enfermeiros, $50 \%$ eram técnicos e $20 \%$ auxiliares de enfermagem; $38,8 \%$, servidores públicos, $56,3 \%$ trabalhavam em plantões noturnos e $47,5 \%$ referiram outro emprego na enfermagem. O escore total do ICT apresentou distribuição normal tanto no teste $(\mathrm{p}=0,587)$, quanto no reteste $(\mathrm{p}=$ $0,237)$, permitindo a realização de análises que levassem em conta a suposição de normalidade, tais como o gráfico de Bland e Altman. Esse gráfico (Figura 1) mostra que $95 \%$ das diferenças entre a $1^{\mathrm{a}}$ e a $2^{\mathrm{a}}$ medidas do ICT apresentaram-se entre - 6 $\mathrm{e}+6$ pontos, com diferenças individuais variando de $-9 \mathrm{a}+8$ pontos no intervalo do estudo; 33 sujeitos obtiveram um escore acima da média e 32 sujeitos abaixo. Foram observados 4 pontos (5\%) fora do limite da média \pm 2 desvios-padrão.

A média dos escores do ICT no teste e no reteste foi semelhante (39,7 pontos [DP $=4,8$ ] versus 39,6 pontos [ $[\mathrm{DP}=5,0]$ ), não sendo essa diferença estatisticamente significativa $\left(0,175\right.$ pontos, $\operatorname{com} \mathrm{IC}_{95 \%}=[-0,535$ a 0,885$])$. O escore total do ICT apresentou $\mathrm{CCI}=0,79\left(\mathrm{IC}_{95 \%} 0,67\right.$ a 0,86$)$. Quando avaliado por item, o ICT mostrou uma concordância, mensurada pelo Kappa ponderado quadrático, que variou de regular $(0,39)$ para os itens "Considerando sua saúde, você acha que será capaz de daqui a 2 anos fazer o seu trabalho atual?" e "Capacidade para o trabalho comparada com a melhor de toda a vida" a quase perfeita $(0,82)$ para os escores das doenças atuais diagnosticadas pelo médico (Tabela 1).

Quando analisado em quatro categorias (ICT categórico), o percentual de concordância foi de $67,5 \%$ (kappa ponderado quadrático $=0,69 ; \mathrm{IC}_{95 \%} 0,50$ a 0,80 ). No reteste, 13 sujeitos foram classificados em uma categoria superior, e 13 em uma categoria inferior, comparados com a classificação da primeira medida (Tabela 2 ). 


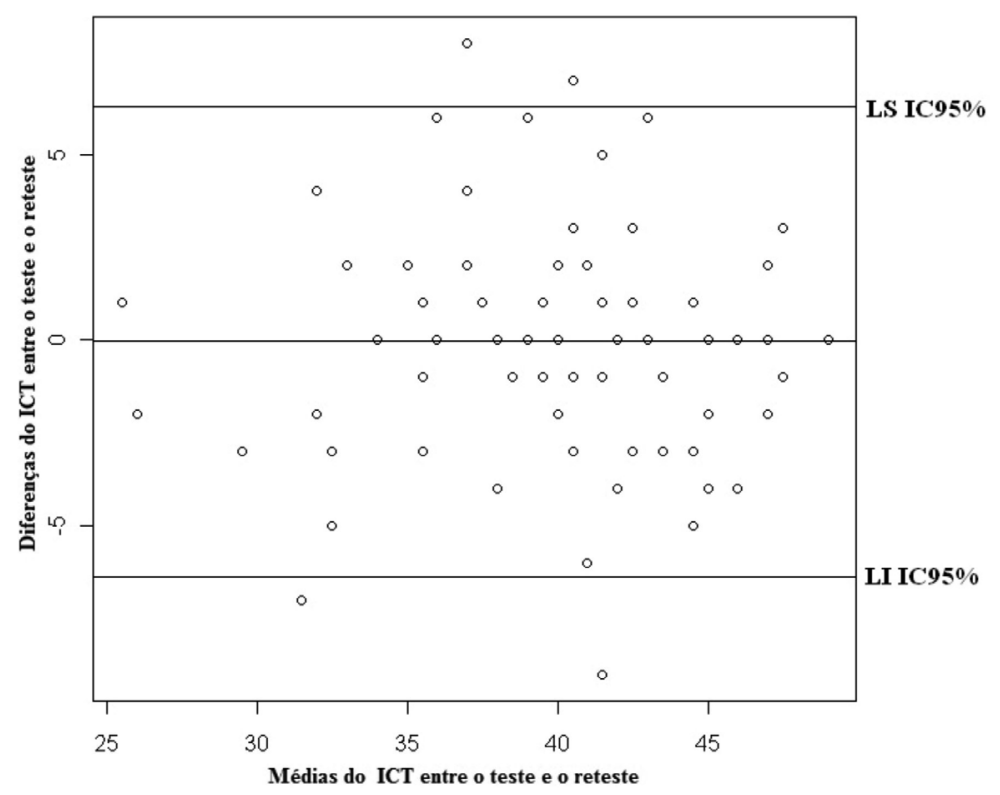

Figura 1 - Gráfico de Bland e Altman, diferenças entre o teste e o reteste contra as médias do teste e reteste e limites dos intervalos de confiança $95 \%$ em trabalhadores de enfermagem do Rio de Janeiro, RJ, 2005 ( $\mathrm{N}=80$ ).

Figure 1 - Bland and Altman plot, differences between test and retest against the mean test and retest and limits of confidence intervals 95\% in nursing in Rio de Janeiro, $R J, 2005(N=80)$.

\section{Discussão}

De maneira geral, o estudo sugere uma adequação de propriedades psicométricas do ICT no que se refere à estabilidade teste-reteste entre trabalhadores de enfermagem. O gráfico de Bland e Altman não mostrou um padrão sistemático, ou seja, as diferenças parecem ser aleatórias; os índices obtidos variaram de concordância regular a quase perfeita, demonstrando confiabilidade teste-reteste aceitável do instrumento.

Resultados semelhantes foram identificados em trabalhadores da construção ${ }^{22}$, em que $5 \%$ dos pontos ficaram fora do limite esperado $(-6,86 \mathrm{a}+6,86)$. Os dados de concordância destes autores também se assemelham aos nossos, já que eles observaram concordância de 66\% (64 de 97), com 13 sujeitos classificados em uma categoria superior em relação à primeira medida $\mathrm{e}$ 19 classificados em uma categoria inferior.

Entretanto, nossos resultados diferem daqueles encontrados por Renosto e colaboradores ${ }^{15}$ em metalúrgicos em que apenas dois pontos $(1,3 \%)$ ficaram fora do limite $(-7,1 \mathrm{a}+7,1$ pontos). Estes autores identificaram CCI para o escore global de 0,84 e o Kappa ponderado variou de 0,54 para o item "Capacidade para o trabalho, comparada com a melhor de toda a vida" até 0,90 para o escore das doenças atuais diagnosticadas pelo médico.

Entre os fatores que podem explicar as diferenças entre os resultados destes estudos estão aspectos metodológicos relativos ao preenchimento do questionário e ao intervalo entre o teste e o reteste. Em nosso estudo, assim como no de Zwart e colaboradores ${ }^{22}$, utilizou-se questionário auto-preenchível, enquanto Renosto e colaboradores ${ }^{15}$ se basearam em aplicação assistida, o que pode contribuir para melhorar o desempenho psicométrico do instrumento. Já o menor intervalo na aplicação do ICT nesse estudo (entre uma e duas semanas), comparado ao intervalo de 4 semanas nos estudos citados, poderia favorecer uma maior concordância, em virtude da maior chance de recordar a resposta fornecida na primeira aplicação. No entanto, este fator não explica os resultados, já que os índices 
Tabela 1 - Coeficiente de Correlação Intraclasse e Kappa ponderado quadrático das dimensões e do escore total do ICT. Table 1 - Interclass correlation coefficient and squared weighted kappa of the dimensions and total score of the WAI.

\begin{tabular}{|c|c|c|c|c|c|c|}
\hline Itens e dimensão do ICT & $\begin{array}{c}\text { Kappa } \\
\text { ponderado } \\
\text { quadrático }\end{array}$ & IC95\% & $\mathrm{CCl}$ & IC95\% & $\begin{array}{l}\text { Média } \\
\text { Teste }\end{array}$ & $\begin{array}{l}\text { Média } \\
\text { Reteste }\end{array}$ \\
\hline $\begin{array}{l}\text { 1. Capacidade para o trabalho comparada } \\
\text { com a melhor de toda vida. }\end{array}$ & 0,39 & $0,16-0,64$ & 0,39 & $0,08-0,63$ & 8,39 & 7,99 \\
\hline $\begin{array}{l}\text { 2. Capacidade para o trabalho em relação } \\
\text { às exigências físicas. }\end{array}$ & 0,65 & $0,50-0,76$ & 0,66 & $0,50-0,77$ & 7,93 & 8,08 \\
\hline $\begin{array}{l}\text { 2.1. Como você classificaria sua } \\
\text { capacidade para o trabalho em relação } \\
\text { às exigências físicas do seu trabalho? }\end{array}$ & 0,54 & $0,39-0,67$ & - & - & & \\
\hline $\begin{array}{l}\text { 2.2. Como você classificaria sua } \\
\text { capacidade para o trabalho em relação } \\
\text { às exigências mentais do seu trabalho? }\end{array}$ & 0,62 & $0,44-0,74$ & - & - & & \\
\hline $\begin{array}{l}\text { 3. Número de doenças atuais } \\
\text { diagnosticadas pelo médico. }\end{array}$ & 0,77 & $0,66-0,86$ & 0,77 & $0,65-0,85$ & & \\
\hline 3.1. Escore das doenças & 0,82 & $0,70-0,90$ & - & - & 4,01 & 4,24 \\
\hline $\begin{array}{l}\text { 4. Sua lesão ou doença é um impedimento } \\
\text { para seu trabalho atual? }\end{array}$ & 0,53 & $0,32-0,72$ & - & - & 5,16 & 5,11 \\
\hline $\begin{array}{l}\text { 5.Quantos dias inteiros você esteve fora } \\
\text { do trabalho durante os últimos } 12 \text { meses? }\end{array}$ & 0,61 & $0,32-0,84$ & - & - & 4,33 & 4,35 \\
\hline $\begin{array}{l}\text { 6. Considerando sua saúde, você acha que } \\
\text { será capaz de daqui a } 2 \text { anos fazer seu } \\
\text { trabalho atual? }\end{array}$ & 0,39 & $0,07-0,74$ & - & - & & \\
\hline 7.Recursos mentais. & 0,74 & $0,57-0,85$ & - & - & & \\
\hline $\begin{array}{l}\text { 7.1. Recentemente você tem conseguido } \\
\text { apreciar suas atividades diárias? }\end{array}$ & 0,54 & $0,35-0,70$ & - & - & & \\
\hline $\begin{array}{l}\text { 7.2. Recentemente você tem se sentido } \\
\text { ativo ou alerta? }\end{array}$ & 0,73 & $0,59-0,83$ & - & - & & \\
\hline $\begin{array}{l}\text { 7.3. Recentemente você tem se sentido } \\
\text { cheio de esperança para o futuro? }\end{array}$ & 0,69 & $0,49-0,82$ & - & - & & \\
\hline Total & - & - & 0,79 & $0,67-0,86$ & 39,7 & 39,6 \\
\hline
\end{tabular}

Tabela 2 - Classificação dos sujeitos, de acordo, com a categoria do ICT nas medidas de teste- reteste.

Table 2 - Classification of subjects according, to the category of ICT in measures of test-retest.

\begin{tabular}{llccccc}
\hline & & \multicolumn{4}{c}{ ICT categórico teste } \\
\cline { 3 - 7 } & & Baixa & Moderada & Boa & Ótima & Total \\
\hline ICT categórico reteste & Baixa & $\mathbf{2}$ & 0 & 0 & 0 & $2(2,5 \%)$ \\
& Moderada & 0 & $\mathbf{1 3}$ & 2 & 1 & $16(20,0 \%)$ \\
& Boa & 0 & 8 & $\mathbf{2 7}$ & 10 & $45(56,3 \%)$ \\
& Ótima & 0 & 0 & 5 & $\mathbf{1 2}$ & $17(21,3 \%)$ \\
& Total & $2(2,5 \%)$ & $21(26,3 \%)$ & $34(42,5 \%)$ & $23(28,8 \%)$ & $80(100 \%)$ \\
Kappa ponderado quadrático & 0,69 & $\mathrm{IC}_{95 \%}$ & $(0,50 \mathrm{a} 0,80)$ & & & \\
\hline
\end{tabular}

de concordância foram semelhantes aos obtidos por Zwart e colaboradores ${ }^{22}$ (auto-preenchido e 4 semanas) e mais baixos do que aqueles obtidos por Renosto e colaboradores $^{15}$ (assistida e 4 semanas). Segundo
Streiner e Norman ${ }^{23}$, o período de aplicação do reteste não deve ser muito curto, pois a pessoa poderia simplesmente se lembrar das respostas, nem muito longo, já que mudanças na ocorrência dos eventos poderiam 
explicar as variações identificadas.

Vale ressaltar que a variação do intervalo teste-reteste (entre 9 e 19 dias) entre os trabalhadores avaliados não afetou o desempenho psicométrico deste estudo. Análises complementares indicam que a amplitude do intervalo do tempo para realização do reteste não introduziu variabilidade capaz de comprometer os resultados deste estudo. Tomando como ponto de corte a mediana do tempo do reteste (12 dias), o teste t para amostras pareadas não mostra diferença significativa entre o escore global do ICT no teste e reteste, comparando os que responderam com intervalo de até 12 dias (Teste $\mathrm{t}=$ $0,643, p=0,522$ ) e aqueles que responderam com intervalo maior que 12 dias (Test $\mathrm{t}=$ $0,465 \mathrm{p}=0,643$ ).

Doze respondentes tiveram um ou dois itens com respostas incompletas, sendo imputados esses valores conforme descrito anteriormente. A imputação tanto pela média quanto pela mediana favorecem a maior concordância, pois reduz a variabilidade dos dados.

Embora o estudo tenha incluído trabalhadores de enfermagem de diferentes características, por meio de amostragem aleatória sistemática, o reduzido tamanho da amostra compromete a precisão das estimativas, além de não permitir explorar a confiabilidade do ICT segundo subgrupos relativos à escolaridade, sexo e idade. No entanto, a amostra é suficiente para estudos de avaliação psicométrica, que recomenda cerca de 10 sujeitos para cada item/dimensão avaliada ${ }^{24,25}$.

Um fator limitante a ser considerado no presente estudo é que o tamanho da amostra não permitiu explorar a confiabilidade do ICT segundo subgrupos relativos à escolaridade, sexo e idade. Além disso, a utilização de um grupo de trabalho específico, como os trabalhadores em enfermagem, não permite generalização do presente estudo para outras ocupações. Outra limitação, já apontada no estudo de Martinez et al. ${ }^{3}$ é a definição dos pontos de corte do escore do ICT, com base em resultados de trabalhadores finlandeses. Como os trabalhadores brasileiros têm diferente composição demográfica e estão expostos a condições de trabalho e de vida diferentes daquelas existentes na Finlândia, possivelmente estão sujeitos a um padrão de envelhecimento funcional diferente e, por isso, os pontos de corte originais podem não ser válidos.

É importante ressaltar o efeito do trabalhador sadio, presente em estudos transversais em epidemiologia ocupacional que, muitas vezes, exclui o possível doente nas pesquisas ${ }^{26,27}$. Este efeito pode levar à subestimativa dos riscos oferecidos pelo processo de trabalho, porque os que são mais afetados não conseguem se manter no emprego, afastando-se por licenças para tratamento de saúde, por demissões e por outros motivos.

Os resultados aceitáveis em relação à estabilidade dão suporte adicional para a aplicabilidade do índice em pesquisas na área de saúde do trabalhador. Novos estudos acerca da validade do ICT em trabalhadores de enfermagem estão sendo realizados pelo mesmo grupo de pesquisa, buscando complementar a avaliação da adaptação psicométrica.

\section{Referências}

1. Tuomi K, Ilmarinen J, Katajarinne L, Tulkki A. Índice de Capacidade para o Trabalho. $1^{\text {a }}$ ed. São Carlos: EDUFSCAR; 2005.

2. Welch LS. Improving work ability in construction workers--let's get to work. Scand J Work Environ Health 2009; 35(5): 321-4.
3. Martinez MC, Latorre M do RD de O, Fischer FM. Validade e confiabilidade da versão brasileira do Índice de Capacidade para o Trabalho. Rev Saúde Pública 2009; 43: 525-32. 
4. Metzner RJ, Fischer FM. Fadiga e capacidade para o trabalho em turnos fixos de doze horas. Rev Saúde Pública [Internet] 2001; 35. Disponível em: http://www.scielosp.org/scielo.php?pid=S003489102001000600008\&script=sci_arttext. [Acessado em 8 de setembro de 2011]

5. Metzner RJ, Fischer FM, Nogueira DP. Comparação da percepção de fadiga e de capacidade para o trabalho entre trabalhadores têxteis de empresas que se encontram em diferentes estágios de responsabilidade social empresarial no estado de São Paulo, Brasil. Saúde Soc 2008; 17: 46-55.

6. Walsh I, Corral S, Franco R, Canetti E, Alem M, Coury H. Capacidade para o trabalho em indivíduos com lesões músculo-esqueléticas crônicas. Rev Saúde Pública 2004; 38: 149-56.

7. Martinez MC, Latorre M do RD de O. Saúde e capacidade para o trabalho em trabalhadores de área administrativaHealth and work ability among office workers. Rev Saúde Pública 2006; 40: 851-8.

8. Marqueze EC, Moreno CR de C. Satisfação no trabalho e capacidade para o trabalho entre docentes universitários. Psicologia em Estudo 2009;14.

9. Sampaio RF, Coelho CM, Barbosa FB, Mancini MC, Parreira VF. Work ability and stress in a bus transportation company in Belo Horizonte, BrazilAvaliação da capacidade para o trabalho e estresse em uma empresa de transporte coletivo de Belo Horizonte, Brasil. Ciênc saúde colet 2009; 14: 287-96.

10. Duran ECM, Cocco MIM. Capacidade para o trabalho entre trabalhadores de enfermagem do prontosocorro de um hospital universitárioWork ability among nursing workers at the emergency service of a university hospital. Habilidad para el trabajo entre trabajadores de enfermería del puesto de socorro de un hospital universitario. Rev Latino-Am Enfermagem 2004; 12: 43-9.

11. Rotenberg L, Griep RH, Fischer FM, Fonseca M de JM, Landsbergis P. Working at night and work ability among nursing personnel: when precarious employment makes the difference. Int Arch Occup Environ Health 2009; 82(7): 877-85.

12. Rotenberg L, Portela LF, Banks B, Griep RH, Fischer FM, Landsbergis P. A gender approach to work ability and its relationship to professional and domestic work hours among nursing personnel. Appl Ergon 2008; 39(5): 64652.

13. Fischer FM, Borges FN da S, Rotenberg L, Latorre M do RD de O, Soares NS, Rosa PLFS et al. Work ability of health care shift workers: What matters? Chronobiol Int 2006; 23(6): 1165-79.

14. Silva Junior SHA da, Vasconcelos AGG, Griep RH, Rotenberg L. Validade e confiabilidade do índice de capacidade para o trabalho (ICT) em trabalhadores de enfermagem. Cad Saúde Pública 2011; 27: 1077-87.
15. Renosto A, Biz P, Hennington ÉA, Pattussi MP. Confiabilidade teste-reteste do Índice de Capacidade para o Trabalho em trabalhadores metalúrgicos do Sul do Brasil. Rev Bras Epidemiol 2009; 12: 217-25.

16. Portela LF. Morbidade referida em profissionais da enfermagem: relações com o horário de trabalho, jornada semanal e trabalho doméstico [dissertação de mestrado]. Rio de Janeiro: Escola Nacional de Saúde Pública; 2003.

17. Peters VPJM, de Rijk AE, Boumans NPG. Nurses' satisfaction with shiftwork and associations with work, home and health characteristics: a survey in the Netherlands. J Adv Nurs 2009; 65(12): 2689-700.

18. Fleiss JL, Cohen J. The Equivalence of Weighted Kappa and the Intraclass Correlation Coefficient as Measures of Reliability. Educational and Psychological Measurement 1973; 33(3): 613-9.

19. Landis JR, Koch GG. The measurement of observer agreement for categorical data. Biometrics 1977; 33(1): 159-74.

20. Bland JM, Altman DG. Statistical methods for assessing agreement between two methods of clinical measurement. Lancet 1986; 1(8476): 307-10.

21. Anderson LF. Legislative roll-call analysis. Evanston: Northwestern University Press; 1966.

22. Zwart BCH, Frings Dresen MHW, van Duivenbooden JC. Test-retest reliability of the Work Ability Index questionnaire. Occupational Medicine 2002; 52(4): 177 81 .

23. Streiner DL, Norman GR. Health Measurement Scales: A practical guide to their development and use. $4^{\text {th }} \mathrm{ed}$. Oxford: Oxford University Press, USA; 2008.

24. Crocker. Introduction to Classical and Modern Test Theory. $1^{\text {st }}$ ed. New York: Wadsworth Publishing; 1986.

25. Pasquali L. Instrumentos psicológicos: manual prático de elaboração. Brasília: LabPAM/IBAPP; 1999.

26. McMichael AJ. Standardized mortality ratios and the "healthy worker effect": Scratching beneath the surface. J Occup Med 1976; 18(3): 165-8.

27. Checkoway H, Pearce N, Crawford-Brown DJ. Research Methods in Occupational Epidemiology. First Edition. New York/Oxford: Oxford University Press, USA; 1989.

Recebido em: 03/03/11

Versão final apresentada em: 15/09/11 Aprovado em: 09/12/11 